\title{
Treatment of Advanced Hepatocellular Carcinoma with Emphasis on Hepatic Arterial Infusion Chemotherapy and Molecular Targeted Therapy
}

\author{
Masatoshi Kudo
}

Department of Gastroenterology and Hepatology, Kinki University School of Medicine, Osaka, Japan

\section{Key Words}

Advanced liver cancer . HAIC combined with sorafenib · Hepatic arterial infusion chemotherapy · Molecular target therapy · Sorafenib

\begin{abstract}
Advanced hepatocellular carcinoma is defined as liver cancer with vascular invasion or extrahepatic metastasis that is untreatable by local therapy. In Japan, hepatic arterial infusion chemotherapy (HAIC) with interferon plus 5-fluorouracil (5-FU) or a combination of low-dose 5-FU and cisplatin, referred to as low-dose FP, is administered for treating advanced liver cancer and yields favorable outcomes. Outside Japan, the molecular targeted agent, sorafenib, is used as a first-line treatment for advanced liver cancer. New drug development for advanced liver cancer and clinical trials on combination therapy with sorafenib and HAIC are currently underway. The prognosis of advanced liver cancer will significantly improve if these clinical trials yield positive results.

Copyright (C) 2012 S. Karger AG, Basel
\end{abstract}

\section{Introduction}

Advanced liver cancer is defined as liver cancer at an advanced stage, which is unresponsive to local treatment and includes hepatocellular carcinoma (HCC) with vascular invasion and liver cancer with distant metastasis. Based on the treatment algorithm proposed by the Japan Society of Hepatology, advanced liver cancer is defined as one that is not eligible for

Masatoshi Kudo, MD, PhD

Department of Gastroenterology and Hepatology, Kinki University School of Medicine 


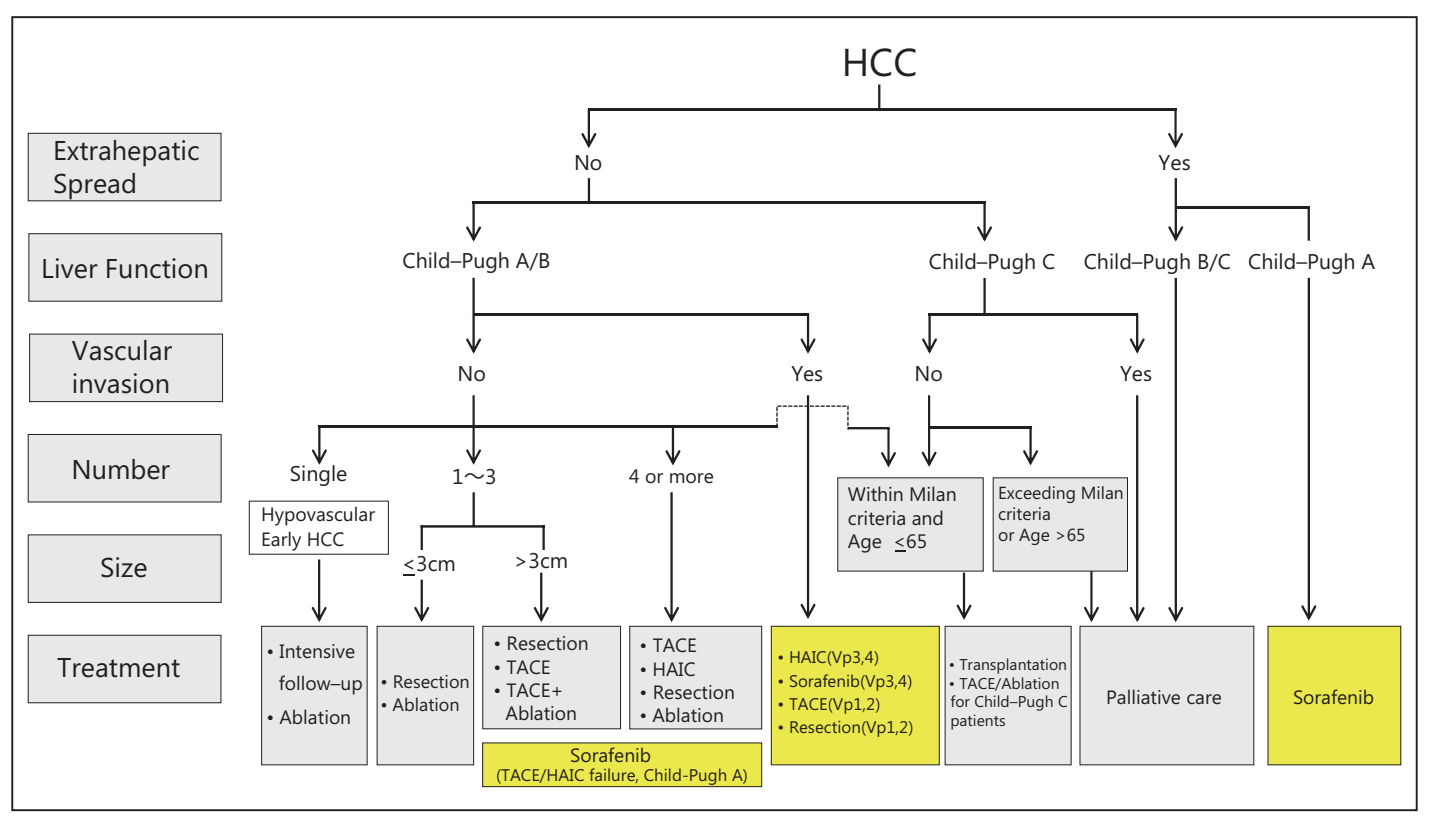

Fig. 1. Consensus-based treatment algorithm for HCC proposed by the Japan Society of Hepatology, revised in 2010. HAIC is recommended for treating multiple liver cancer and HCC with vascular invasion. Sorafenib is recommended for patients unresponsive to TACE and for those with liver cancer with vascular invasion or extrahepatic spread. Modified with permission from Kudo et al. [1].

curative treatment, transcatheter arterial chemoembolization (TACE), or equivalent treatment [1]. The Barcelona Clinic Liver Cancer (BCLC) classification system, used commonly in Western countries defines grade A as early HCC, grade B as intermediate stage, grade C as advanced stage, and grade D as terminal stage [2]. Terminal-stage disease corresponds to Child-Pugh grade C HCC. Therefore, advanced liver cancer corresponds to BCLC grade C cancer, which that is graded as Child-Pugh A or B with vascular invasion or distant metastasis. In Japan, cancers that are unlikely to respond to locoregional therapies such as resection, local ablation, or TACE are defined as advanced liver cancer. These include multiple liver cancer with 4 or more lesions and liver cancer with vascular invasion or distant metastasis.

\section{Treatment}

\section{Systemic chemotherapy}

As described in the American Association for the Study of Liver Diseases (AASLD) guidelines for liver cancer [2], systemic chemotherapy has not been shown to provide any survival benefit, and thus, is not recommended or used in practice. Therefore, this article does not discuss systemic chemotherapy.

\section{Hepatic arterial infusion chemotherapy}

In Japan, hepatic arterial infusion chemotherapy (HAIC) is primarily administered for treating HCC with vascular invasion. Based on the Japanese HCC management guideline [1, 3], HAIC is indicated and recommended for treating the following: multiple liver cancer with 4 or more lesions, HCC with major vascular invasion, and cancers with distant metastasis not considered a prognostic factor (fig. 1). According to the 18th nationwide survey of the Liver 
Fig. 2. Treatment modality of initially diagnosed HCC in Japan. Only $5 \%$ patients received chemotherapy [19].

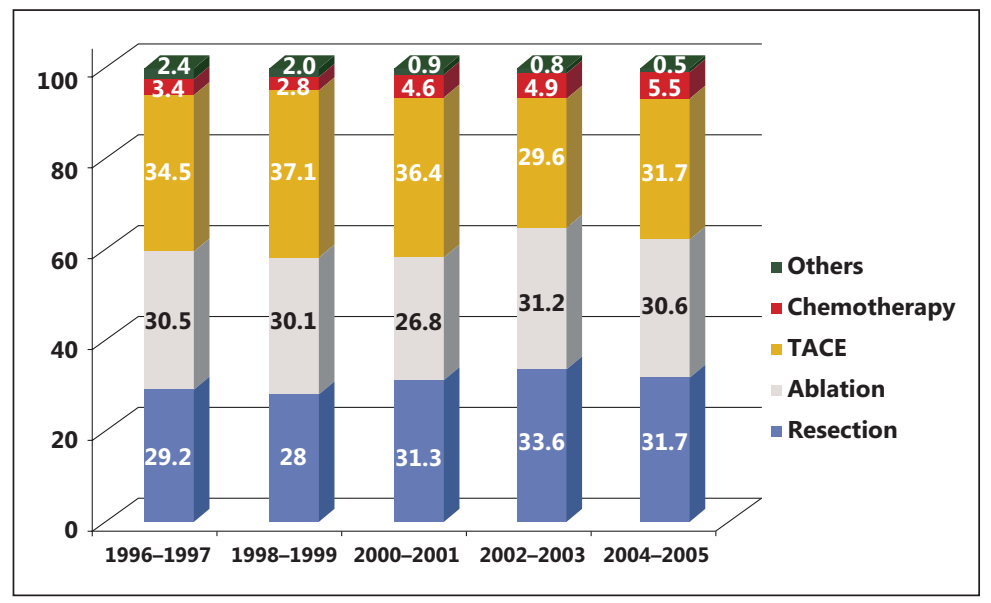

Fig. 3. Administration method of chemotherapeutic agent. Nearly $90 \%$ patients received HAIC [19].

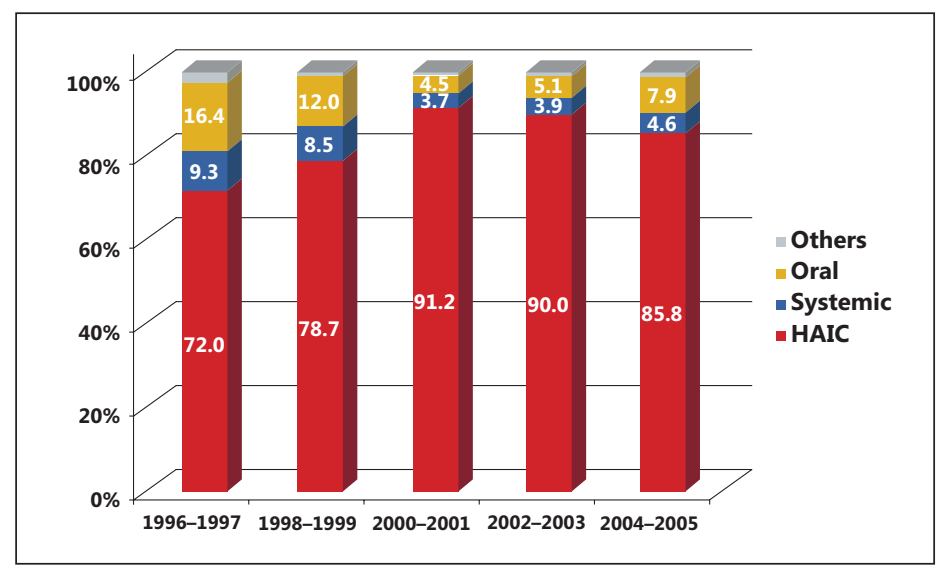

Cancer Study Group of Japan, chemotherapy is performed only in 5.5\% patients with liver cancer (fig. 2), and approximately 90\% of these patients undergo HAIC [4](Fig. 3). In addition, chemotherapy demonstrated an excellent outcome in a nationwide survey, with a response rate (complete response (CR) plus partial response (PR) of $45.9 \%$ and a disease control rate of 76.5\% (fig. 4). In Japan, the interferon (IFN) + 5-fluorouracil (5-FU), low-dose FP (low-dose 5-FU + cisplatin (CDDP)), and CDDP monotherapy regimens account for 49\%, 30\%, and 11\% of all HAIC regimens, respectively (fig. 5). HAIC has also demonstrated a favorable outcome in our study, with a median survival time (MST) of 15.9 months and a time to progression (TTP) of 4.1 months [5](Figs. 6a and 6b). A subset of patients who responded to the therapy (CR + PR) exhibited MST of 40.7 months, while those who did not respond to the therapy (SD + PD) exhibited MST of 6.8 months, suggesting a better survival among responders than among nonresponders (fig. 6c). These results indicate that HAIC is superior to other treatments. Propensity analysis of the nationwide survey clearly revealed that HAIC is superior to the best supportive care based on a nationwide survey of Liver Cancer Study Group of Japan (fig. 7).

HAIC was developed in Japan but is not appreciated in Western countries because no randomized controlled trial (RCT) has been conducted and its use is based solely on empirical data. In fact, AASLD clinical practice guidelines for liver cancer strongly recommend that HAIC should not be used as the standard of care [6]. To overcome this obstacle, there is a strong need to conduct RCTs in Japan. However, since the effectiveness of empirically established 


\section{Liver Cancer}

Fig. 4. Result of HAIC in Japan. The response rate is $45.9 \%$ and disease control rate is $76.5 \%$ [19].

\begin{tabular}{l|l}
\hline \multicolumn{2}{l}{ Liver Cancer 2012;1:62-70 } \\
\hline DOI: $10.1159 / 000342402$ & $\begin{array}{l}\text { C } 2012 \text { S. Karger AG, Basel } \\
\text { www.karger.com/lic }\end{array}$ \\
Published online: September 28, 2012 &
\end{tabular}

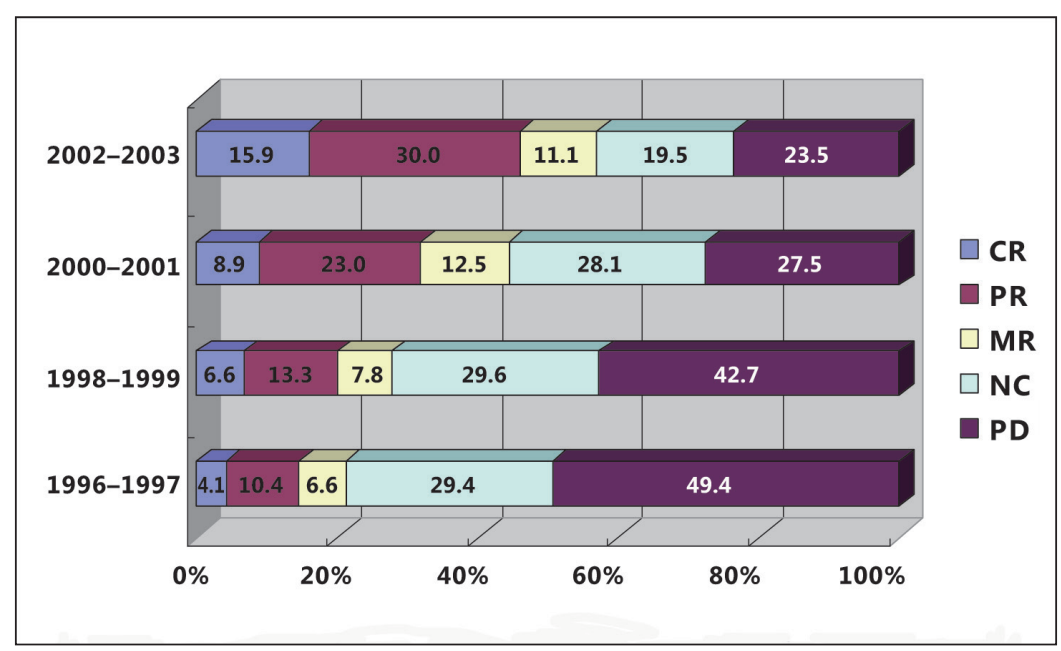

Fig. 5. Most commonly used HAIC regimens in Japan. IFN + 5-FU regimen followed by low-dose FP therapy is the most popular.

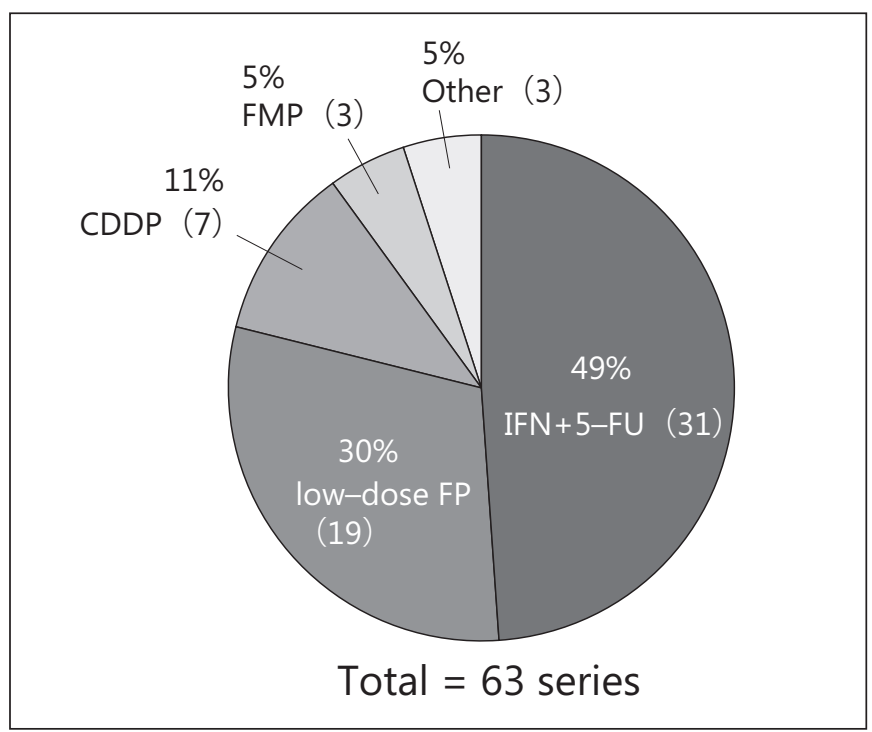

HAIC has been widely accepted by Japanese physicians and patients, the conduct of RCTs has been precluded by ethical concerns. This situation resembles that encountered in the history of TACE [7], which was also empirically developed in Japan but had not been approved until RCTs conducted in the West or East including Japan confirmed its efficacy and safety.

An advantage of HAIC is that the response can be estimated on the basis of tumor markers, imaging findings, or other parameters 2-4 weeks after therapy. Consequently, both responders and nonresponders can be distinguished from each other in less than 4 weeks. For nonresponders, treatment can be switched to a molecular targeted therapy such as sorafenib, a global standard of care for advanced HCC. There is a growing argument about whether HAIC or molecular targeted therapy should be selected as the first choice of treatment for liver cancers with vascular invasion, for patients not eligible for TACE therapy, and for those with multiple liver cancers unresponsive to TACE. HAIC generally provides good but variable outcomes, with a response rate of 8-71\% and MST of 6-15.9 months. A finding that was similar to previous reports was that responders demonstrated better outcome than nonresponders. Considering this, HAIC will remain an essential treatment option for advanced liver cancer if used appropriately. 


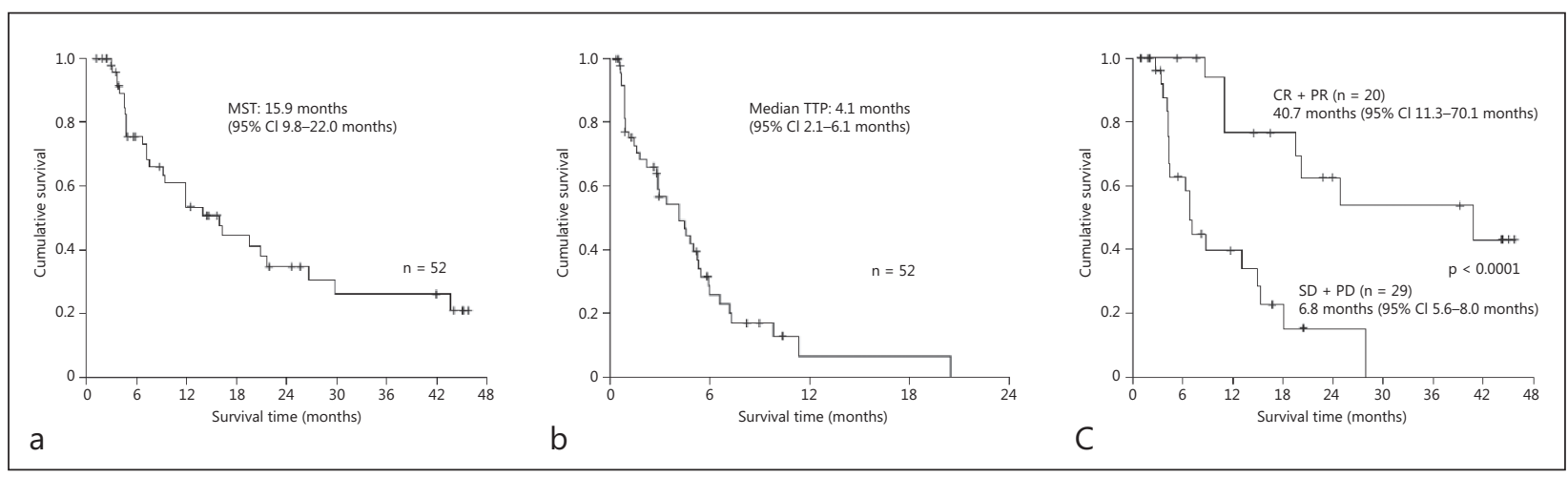

Fig. 6 a Kaplan-Meier analysis of overall survival of 52 patients treated by HAIC with low-dose FP therapy. b KaplanMeier analysis of time to progression in 52 patients treated by HAIC with low-dose FP therapy. The median time to progression was 4.1 months (95\% CI 2.1-6.1). c The median survival for patients who underwent HAIC with low-dose FP therapy at Kinki University and who responded to the therapy (responders) was 40.7 months, which was significantly longer than nonresponders (6.8 months). Reproduced with permission from Ueshima, et al. [20]

Fig. 7. Survival of patients with HCC with vascular invasion and/or extrahepatic spread and Child-Pugh A/B: transcatheter arterial chemoembolization and HAIC after case matching according to propensity score analysis. Number of patients before case matching in HAIC and BSC was 824 and 1,345, respectively.

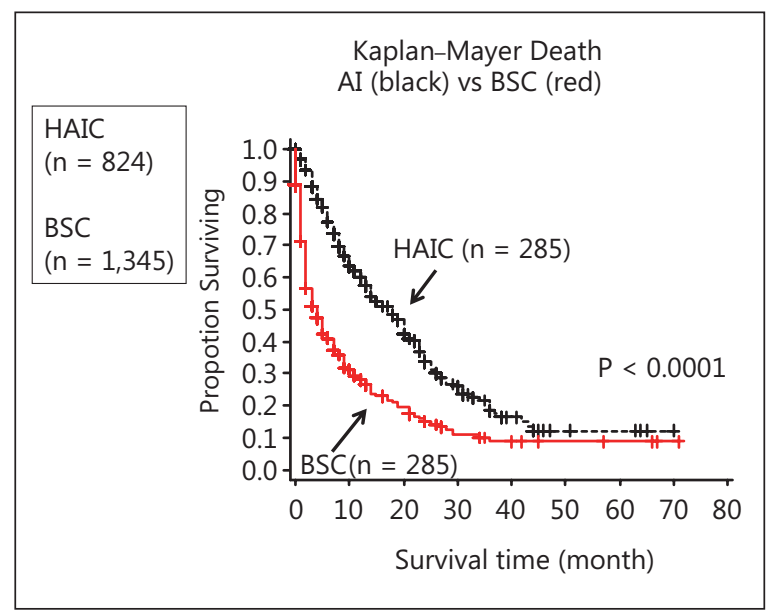

\section{Molecular targeted therapy}

1) Mechanism of action and effects of sorafenib

Sorafenib is an oral molecular targeted agent that has been demonstrated to be effective for treating HCC for the first time and is currently the only insurance-covered drug. The Raf/ MEK/ERK pathway, the so-called MAP kinase pathway, is a downstream pathway shared by VEGFR, PDGFR, and EGFR, and its activation has been demonstrated to be involved in the growth and survival of HCC. Sorafenib inhibits HCC growth by suppressing the serine/threonine kinase activity of the C-Raf and B-Raf components of the MAP kinase pathway and inhibiting FLT-3 and c-KIT activities. It also serves as a multikinase inhibitor that suppresses the tyrosine kinase activity of VEGFR and PDGFR, thereby inhibiting angiogenesis and the growth of endothelial cells and pericytes [8-10].

In a global phase III trial conducted in the West (SHARP study) [11], the median survival time was significantly improved in the sorafenib group compared with the placebo group (10.7 vs. 7.9 months, respectively; hazard ratio (HR): 0.69, $\mathrm{P}<0.001$ ), and the 1 year survival rate also improved from 33 to $44 \%$. The median TTP significantly improved in the sorafenib group (5.5 months) compared with the placebo group (2.8 months, HR: 0.58, P < 0.001). In 
an Asia-Pacific study [12] conducted in China, Taiwan, and North Korea, the median survival time significantly improved in the sorafenib group compared with the placebo group (6.5 vs. 4.2 months, respectively; HR: $0.68, \mathrm{P}=0.014$ ) and TTP (2.8 vs. 1.4 months, respectively; HR: $0.57, \mathrm{P}=0.0005$ ). In Japan, sorafenib was approved for insurance coverage in May 2009 after a phase I clinical trial confirmed its pharmacokinetics and safety [13].

2) Indications of sorafenib

The current indications of sorafenib are specified and described in the consensus-based treatment algorithm proposed by the Japan Society of Hepatology [1] (Fig. 1). These include patients with Child-Pugh grade A HCC with extrahepatic spread or vascular invasion and those not responding to TACE or HAIC. Sorafenib is not recommended for Child-Pugh grade B HCC because of the lack of safety data. It is important to identify unresponsiveness to TACE at the earliest and administer sorafenib before repeated courses of TACE cause impaired liver function or before the disease stage progresses beyond Child-Pugh A.

3) Future prospects

(1) Molecular targeted therapy combined with conventional therapy

a. Prevention of recurrence after curative treatment

Ongoing clinical trials aimed at preventing recurrence include the Sorafenib as Adjuvant Treatment in the Prevention of Recurrence of HCC (STORM) study of sorafenib and another study of peretinoin (NIK333), an acyclic retinoid. The STORM study is a global trial being conducted in Europe, North America, South America, and Asian countries, including Japan, to evaluate the preventive effect of sorafenib on recurrence in patients who have undergone radio frequency ablation or resection. Patient enrollment has been completed, and the study is currently in the follow-up period.

NIK333 (peretinoin) is an oral acyclic retinoid developed in Japan, which has a vitamin A-like structure and primarily targets the nuclear receptors of retinoid, namely retinoic acid receptor (RAR) and retinoid X receptor (RXR). This agent exerts transcriptional activationand differentiation-inducing effects via RAR and RXR and is expected to prevent carcinogenesis through HCC precursor removal by apoptosis and differentiation induction [14]. A phase III clinical trial to establish the superiority of peretinoin $600 \mathrm{mg}$ over placebo is currently underway.

b. Post-TACE adjuvant therapy

The majority of patients with HCC receive at least 1 cycle of TACE. In many cases TACE is repeated because it is not curative and is inevitably followed by recurrence. Although TACE has an antitumor effect, repeated use of this therapy causes gradual deterioration of liver function. Therefore, the adjuvant use of molecular targeted therapy is attracting attention. Although tumor shrinkage is observed following TACE, recurrence, including tumor regrowth and intrahepatic metastasis, can still occur [7]. A suggested mechanism of post-TACE recurrence is mediated by increased angiogenesis in response to increased VEGF levels caused by embolization-induced tumor hypoxia $[15,16]$. Therefore, the suppression of angiogenesis following TACE is important to prevent post-TACE tumor recurrence. The combined use of TACE and angiogenesis inhibitors strongly suppresses post-TACE recurrence or regrowth of advanced HCC through the inhibitory effect of angiogenesis inhibitors on post-TACE increase in angiogenesis. Such a therapy is also expected to prolong the period during which tumor progression can be controlled by TACE and prevent deterioration of liver function by reducing the frequency of TACE. Although a study conducted in Japan and South Korea, the so-called post-TACE study, had various limitations [17], many clinical trials on molecular targeted therapy as a post-TACE-adjuvant or TACE-combination regimen are being conducted on the basis of these study results. 


\section{c. TACTICS study}

The Transcatheter Arterial Chemoembolization Therapy in Combination with Sorafenib (TACTICS) study is a phase II clinical trial on TACE combined with sorafenib conducted in Japanese patients with unresectable HCC. The TACE regimen used in this study is based on actual clinical practice in Japan, which comprises Gelpart as an embolization agent and epirubicin hydrochloride or miriplatin as an anticancer agent. It is performed as on-demand TACE and administered after a certain degree of tumor growth has been observed. The study is designed to include patients with HCC untreatable by surgical resection or percutaneous ablation therapy. After undergoing TACE, patients will be randomized at a 1:1 ratio to receive or not receive oral sorafenib. The primary endpoint is time to untreatable progression, at which point TACE can no longer be performed. This study is currently enrolling patients.

\section{d. BRISK-TA study (brivanib)}

Brivanib is a kinase inhibitor that selectively inhibits VEGFR and FGFR. A phase III clinical trial that compares brivanib with placebo as an adjuvant therapy for TACE is underway.

e. ORIENTAL study (TSU68: orantinib)

TSU68 (orantinib) is a low-molecular weight oral angiogenesis inhibitor that inhibits VEGFR, PDGFR, and FGFR [18]. A phase III clinical trial that evaluates the superiority of combined use of TSU68 over placebo in terms of overall survival in patients with advanced HCC who have undergone TACE is currently underway.

f. Sorafenib combined with HAIC

In Japan, HAIC is initially performed for advanced HCC with major vascular invasion. Without established evidence, HAIC is accepted as a de facto standard and is rarely used outside Japan. Although technically complicated and invasive, this therapy provides excellent local control, and is thus considered effective. Several clinical trials that are evaluating the efficacy of combination therapy with HAIC and sorafenib for treating advanced HCC are currently underway. These include the SILIUS trial (NCT01214343), a randomized phase III study on sorafenib combined with HAIC and low-dose FP using a reservoir system aiming to demonstrate the additional benefits of HAIC. SILIUS is part of a multicenter cooperative study funded by the Ministry of Health, Labour and Welfare of Japan. The primary endpoint for this study is overall survival. Demonstration of additional benefits will be the first indirect demonstration of the survival benefit of low-dose FP therapy. The results of the phase I study were promising, demonstrating the additive effect of HAIC and sorafenib. The CDDP-Sor-rP2 study (UMIN000005703) is a randomized phase II study of sorafenib combined with a one-shot HAIC with CDDP. Similar to the SILIUS study, the CDDP-Sor-rP2 study aims to demonstrate additional benefits of HAIC without the use of a reservoir.

(2) Status of development of new drugs other than sorafenib

As summarized in fig. 8, various first-line and second-line therapies, combination therapies with HAIC/TACE and sorafenib, and adjuvant therapies after curative treatment are currently under investigation as potential new treatment strategies for patients with advanced liver cancer unresponsive or intolerable to sorafenib.

\section{Conclusions}

Since the effectiveness of sorafenib was confirmed, systemic chemotherapy with molecular targeted agents has established its position in the treatment of HCC, which otherwise had to rely on local therapy. This has finally allowed discussion of medication therapy for 


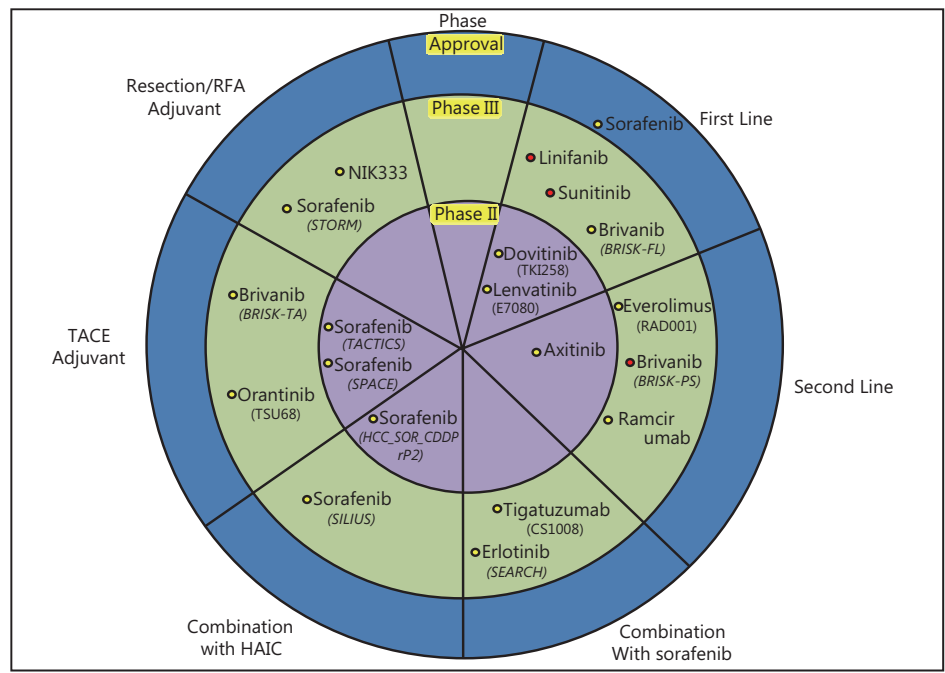

Fig. 8. Current development status of molecular targeted agents

HCC, similar to that for other types of carcinoma. At the same time, it must be remembered that HCC occurs in patients with underlying chronic liver diseases and is accompanied by concomitant conditions such as impaired liver function and pancytopenia. Drugs that cause severe adverse events or affect liver function cannot be used as therapeutic agents, even if they have a potent antitumor effect. The most important challenge for improving the survival outcome in HCC patients is determining ways to prevent deterioration of liver function due to underlying chronic liver disease. The development of less toxic drugs and treatment regimens is therefore desirable.

The prognosis of advanced liver cancer can significantly improve if positive results are obtained from new drug development and clinical studies on combination therapy with existing treatments.

\section{References}

1 Kudo M, Izumi N, Kokudo N, Matsui O, Sakamoto M, Nakashima O, et al: Management of hepatocellular carcinoma in Japan: Consensus-Based Clinical Practice Guidelines proposed by the Japan Society of Hepatology (JSH) 2010 updated version. Dig Dis 2011;29:339-364.

2 Bruix J, Sherman M: Management of hepatocellular carcinoma: An update. Hepatology 2011;53:10201022.

3 Ricke J, Seidensticker M, Mohnike K: Noninvasive diagnosis of hepatocellular carcinoma in cirrhotic liver: Current guidelines and future prospects for radiological imaging. Liver Cancer 2012;1:51-58.

4 Ikai I, Kudo M, Arii S, Omata M, Kojiro M, Sakamoto M, et al: Report of the 18th Follow-up Survey of Primary Liver Cancer in Japan. Hepatol Res 2010;40:1043-1059.

5 Ueshima K, Kudo M, Takita M, Nagai T, Tatsumi C, Ueda T, et al: Des-gamma-carboxyprothrombin may be a promising biomarker to determine the therapeutic efficacy of sorafenib for hepatocellular carcinoma. Dig Dis 2011;29:321-325.

6 Bruix J, Sherman M: Management of hepatocellular carcinoma. Hepatology 2005;42:1208-1236.

7 Lencioni R: Chemoembolization in patients with hepatocellular carcinoma. Liver Cancer 2012;1:41-50.

8 Wilhelm SM, Carter C, Tang L, Wilkie D, McNabola A, Rong H, et al: BAY 43-9006 exhibits broad spectrum oral antitumor activity and targets the RAF/MEK/ERK pathway and receptor tyrosine kinases involved in tumor progression and angiogenesis. Cancer Res 2004;64:7099-7109.

9 Liu L, Cao Y, Chen C, Zhang X, McNabola A, Wilkie D, et al: Sorafenib blocks the RAF/MEK/ERK pathway, inhibits tumor angiogenesis, and induces tumor cell apoptosis in hepatocellular carcinoma model PLC/ PRF/5. Cancer Res 2006;66:11851-11858.

10 Chang YS, Adnane J, Trail PA, Levy J, Henderson A, Xue D, et al: Sorafenib (BAY 43-9006) inhibits tumor growth and vascularization and induces tumor apoptosis and hypoxia in RCC xenograft models. Cancer Chemother Pharmacol 2007;59:561-574. 
11 Llovet JM, Ricci S, Mazzaferro V, Hilgard P, Gane E, Blanc JF, et al: Sorafenib in advanced hepatocellular carcinoma. N Engl J Med 2008;359:378-390.

12 Cheng AL, Kang YK, Chen Z, Tsao CJ, Qin S, Kim JS, et al: Efficacy and safety of sorafenib in patients in the Asia-Pacific region with advanced hepatocellular carcinoma: a phase III randomised, double-blind, placebo-controlled trial. Lancet Oncol 2009;10:25-34.

13 Furuse J, Ishii H, Nakachi K, Suzuki E, Shimizu S, Nakajima K: Phase I study of sorafenib in Japanese patients with hepatocellular carcinoma. Cancer Sci 2008;99:159-165.

14 Okita K, Matsui O, Kumada H, Tanaka K, Kaneko S, Moriwaki H, et al. Effect of peretinoin on recurrence of hepatocellular carcinoma (HCC): Results of a phse II/III randomized placebo-controlled trial. (Abstract \#4024), ASCO, June, 2010.

15 Li X, Feng GS, Zheng CS, Zhuo CK, Liu X: Expression of plasma vascular endothelial growth factor in patients with hepatocellular carcinoma and effect of transcatheter arterial chemoembolization therapy on plasma vascular endothelial growth factor level. World J Gastroenterol 2004;10:2878-2882.

16 Sergio A, Cristofori C, Cardin R, Pivetta G, Ragazzi R, Baldan A, et al: Transcatheter arterial chemoembolization (TACE) in hepatocellular carcinoma (HCC): the role of angiogenesis and invasiveness. Am J Gastroenterol 2008;103:914-921.

17 Kudo M, Imanaka K, Chida N, Nakachi K, Tak WY, Takayama T, et al: Phase III study of sorafenib after transarterial chemoembolisation in Japanese and Korean patients with unresectable hepatocellular carcinoma. Eur J Cancer 2011;47:2117-2127.

18 Laird AD, Vajkoczy P, Shawver LK, Thurnher A, Liang C, Mohammadi M, et al: SU6668 is a potent antiangiogenic and antitumor agent that induces regression of established tumors. Cancer Res 2000;60:4152-4160.

19 Report of the 18th follow-up survey of primary liver cancer in Japan. The Liver Cancer Study Group of Japan, eds.,Media Planning Inc., Japan, 2009.

20 Ueshima K, Kudo M, Takita M, Nagai T, Tatsumi C, Ueda T, Kitai S, Ishikawa E, Yada N, Inoue T, Hagiwara S, Minami Y, Chug H: Hepatic arterial infusion chemotherapy using low-dose 5-fluorouracil and cisplatin for advanced hepatocellular carcinoma. Oncology 2010;78:S148-S153. 